\title{
Targeted treatment of chronic myeloid leukemia: role of imatinib
}

\author{
Ila Tamascar \\ Jeyanthi Ramanarayanan
}

Department of Medical Oncology, Roswell Park Cancer Institute, Elm and Carlton Streets, Buffalo, NY, USA

Correspondence: Jeyanthi Ramanarayanan Assistant Professor, Department of Medical Oncology, Roswell Park Cancer Institute, Elm and Carlton Streets, Buffalo, NY, I4263, USA

Email jeyanthiram88@hotmail.com

\begin{abstract}
Chronic myeloid leukemia (CML) is a myeloproliferative disorder characterized by clonal expansion of pleuripotent hematopoetic stem cells. The incidence of CML is 1 to 2 cases per 100,000 people per year; in the Western Hemisphere, CML accounts for 15\% of leukemias in adults. Discovery of the specific karyotypic abnormality of the Philadelphia $(\mathrm{Ph})$ chromosome in the pathogenesis of CML has led to a better understanding of the disease and hence to an advancement of targeted therapeutics. Availability of imatinib as an accepted targeted therapy in newly diagnosed patients has changed the treatment paradigm in CML. The majority of CML patients in chronic phase achieve excellent and durable responses with standard-dose imatinib. Mechanisms of primary and secondary resistance to imatinib in CML have been extensively studied and newer tyrosine kinase inhibitors are now being evaluated for clinical use. It is important that at any time the CML treatment and response remain optimal and thus patients on imatinib require continuous monitoring for early detection of resistance. This review will discuss the treatment and guidelines for monitoring CML patients in the imatinib era.
\end{abstract}

Keywords: BCR-ABL, imatinib, tyrosine kinase inhibitors, leukemia, CML

Chronic myeloid leukemia (CML) is a myeloproliferative disorder characterized by clonal expansion of pleuripotent hematopoetic stem cells leading to accumulation of cells of myeloid lineage in the peripheral blood, bone marrow and extramedullary sites. In the chronic phase, the progenitor cells retain their ability to differentiate to myeloid lineage cells, but as the disease progresses to accelerated phase and blast crisis (AP and BC) differentiation is suppressed. The incidence of CML is 1 to 2 cases per 100,000 people per year; in the Western hemisphere, CML accounts for $15 \%$ of leukemias in adults. For the year 2008 it is estimated that about 4,830 new cases of CML will be diagnosed in the United States. ${ }^{1}$ Discovery of the specific abnormality of the karyotype, the Philadelphia $(\mathrm{Ph})$ chromosome ${ }^{2}$ linked to the pathogenesis of CML, has led not only to a better understanding of the disease but to advancement of targeted therapeutics and cure.

The $\mathrm{Ph}$ chromosome, the result of a $\mathrm{t}(9 ; 22)$ reciprocal translocation, is identified in over $90 \%$ of patients with chronic myeloid leukemia (CML). ${ }^{3}$ The translocation results in the juxtaposition of DNA sequences from the $B C R$ and $A B L$ genes, ${ }^{4,5}$ which encodes the oncoprotein, p210BCR-ABL. The dysregulated tyrosine kinase activity of BCR-ABL as a result of the gene translocation is essential for leukemogenesis in CML. Identification of the BCR-ABL tyrosine kinase, the product of the chimeric gene produced by the $\mathrm{Ph}$ chromosome as the molecular abnormality in CML, has enabled the development of targeted kinase inhibitors which have revolutionized the treatment of the disorder.

In the laboratory, infection of murine models with retrovirus encoding P210bcr/abl led to the development of CML. ${ }^{6}$ It was then validated that $B C R-A B L$ alone is sufficient 
to cause CML in animal models. ${ }^{7}$ Further mutational analysis has demonstrated that the constitutive activation of tyrosine kinase protein is required for oncogenic activity and leukemogenesis in CML. ${ }^{6,7}$ Recognition of the tyrosine kinase (TK) activity of the Bcr-Abl proteins led to the discovery of a new series of compounds targeted against BCR-ABL-encoded proteins, which inhibited the TK activity (Figure 1), thus aborting the signals controlling the leukemic phenotype. It was hypothesized that an inhibitor of the BCR-ABL tyrosine kinase should be an effective and selective treatment for CML. To this end, STI571 (4-[(4-methyl-1-piperazinyl)methyl]-N-[4-methyl-3-[[4(3-pyridinyl)-2-pyrimidinyl] amino] phenyl] benzamide methanesulfonate (imatinib, Gleevec ${ }^{\circledR}$; Novartis, Basel, Switzerland), a potent and selective inhibitor of the tyrosine kinase activity of BCR-ABL, ${ }^{8}$ was studied in early phase I studies in patients with CML in the chronic phase. High response rates were observed among those treated with daily doses of $300 \mathrm{mg}$ or more and therapy with STI571 was also well tolerated. Due to relatively specific biochemical activity, high response rates, acceptable pharmacokinetics and minimal toxicity profile, imatinib was rapidly introduced into clinical practice.

Imatinib is a small-molecule drug which inhibits the BCR-ABL tyrosine kinase by competitively binding at the ATP-binding site and blocking the downstream signaling pathways necessary for leukemogenesis. ${ }^{8}$ Imatinib has now become standard treatment for newly diagnosed CML and has created a paradigm shift for targeted management of cancer in general. This review will discuss the role of imatinib in the management of CML, guidelines for monitoring and follow up of patients on imatinib, and future challenges associated with tyrosine kinase inhibition in CML.

\section{Management of newly diagnosed CML}

The majority of patients in the Western Hemisphere are diagnosed in the chronic phase of CML. Imatinib mesylate has become the standard of care for treatment of newly

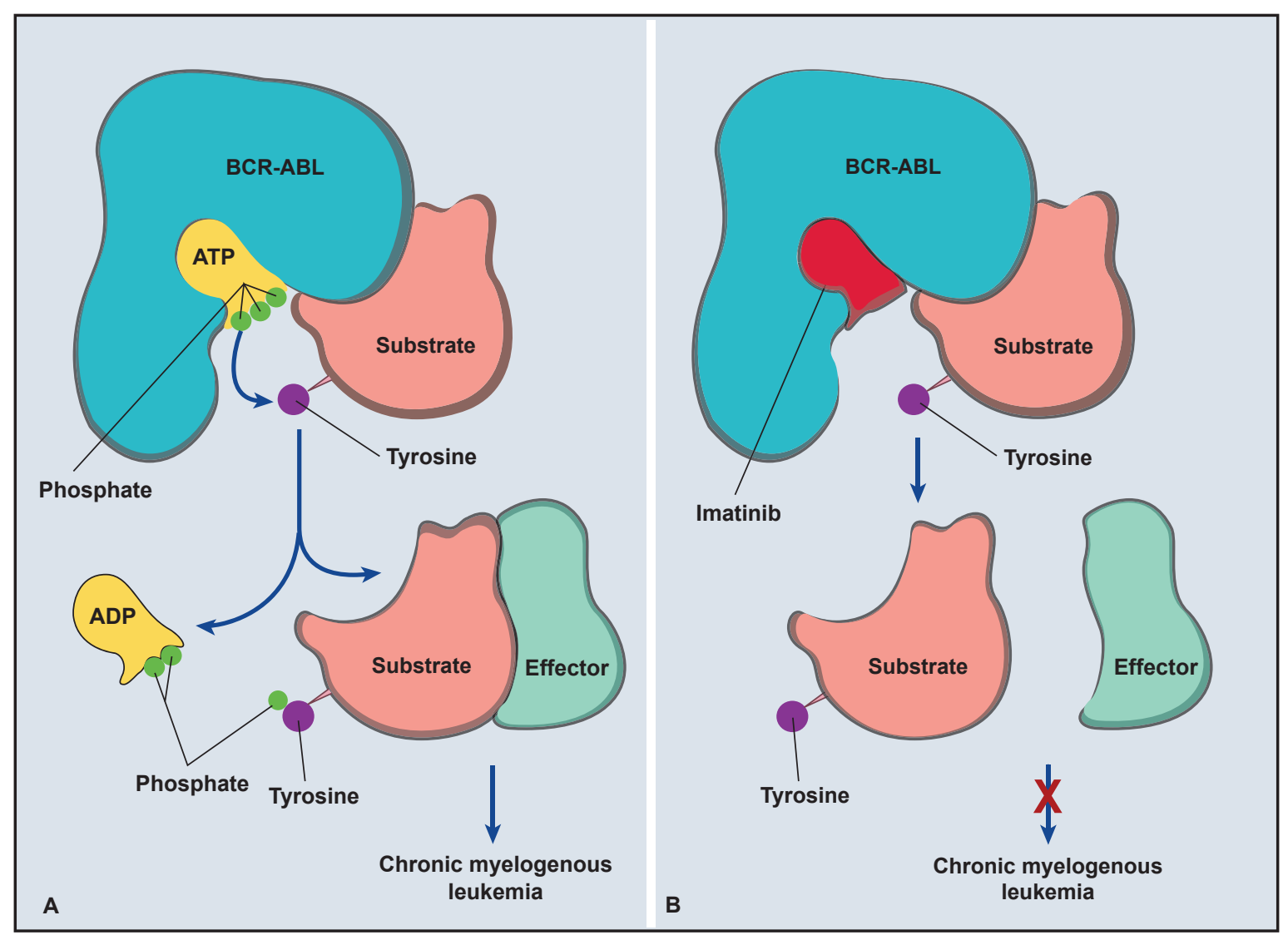

Figure I Mechanism of action of imatinib. A) The phosphorylation and activation of tyrosine residue after binding of adenosine triphosphate ATP in the kinase domain on the BCR-ABL oncoprotein. B) Prevention of phosphorylation and activation of tyrosine residue when imatinib binds to the kinase domain. Adapted with permission from Savage DG, Antman KH. Imatinib mesylate - a new oral targeted therapy. N Engl J Med. 2002;346:683-693."1 Copyright @ 2002 Massuchusetts Medical Society. All rights reserved. 
diagnosed patients with CML. More than half of patients with late chronic-phase CML after failure of interferon alfa-based therapy and over $80 \%$ of newly diagnosed patients achieve a complete cytogenetic response. ${ }^{9}$ Based on the IRIS study data, in December 2002, the Food and Drug Administration (FDA) granted approval for imatinib as frontline treatment for patients with CML.

\section{Definition of responses to imatinib}

The standardized criteria for responses among patients with CML on treatment is as follows. ${ }^{10}$

\section{Hematologic response}

Complete: Defined as platelet count $<450 \times 10^{9} / \mathrm{L}$; white blood cell count $<10 \times 10^{9} / \mathrm{L}$; differential without immature granulocytes and with less than $5 \%$ basophils; nonpalpable spleen.

Partial: Defined as above except for presence of immature cells, platelet count $<50 \%$ of pretreatment but $>450 \times 10^{9} \mathrm{~L}$, persistent splenomegaly but $<50 \%$ pretreatment size.

\section{Cytogenetic response}

Complete: $\mathrm{No} \mathrm{Ph}^{+}$metaphases

Major: $0 \%$ to $35 \% \mathrm{Ph}^{+}$metaphases (complete + partial)

Partial : $1 \%$ to $35 \% \mathrm{Ph}^{+}$metaphases

Minor: $35 \%$ to $90 \% \mathrm{Ph}^{+}$metaphases

None: $\mathrm{Ph}^{+}>95 \%$

\section{Molecular response}

Complete: bcr-abl mRNA transcript nonquantifiable and nondetectable by RT-PCR.

Major: more than or equal to $3 \log$ reduction of bcr-abl mRNA transcript.

\section{Standard dose imatinib}

A dose of $300 \mathrm{mg}$ orally (PO) daily was sufficient to achieve a CHR in almost all chronic phase CML patients, but standard dose which is $400 \mathrm{mg}$ daily is well explored and appears to be easily tolerated. Doses lower than $400 \mathrm{mg}$ are discouraged and cautioned for the possible development of resistance. ${ }^{11}$ The superiority of $400 \mathrm{mg}$ imatinib PO daily over recombinant interferon alfa and low-dose cytarabine was confirmed in a prospective randomized international study (International Randomized Study of Interferon and STI571 [IRIS]). ${ }^{12}$ In this phase III clinical trial, 1106 patients were randomized to imatinib at a dose of $400 \mathrm{mg}$ daily or interferon alfa plus cytarabine. At a follow up around 60 months, imatinib was superior to recombinant interferon alfa for efficacy, with a complete hematologic response rate of $95 \%$ versus $55 \%$, a complete cytogenetic response rate of $76 \%$ versus $15 \%$ and progression-free survival (PFS; survival free from progression to $\mathrm{AP} / \mathrm{BC}$ ) at 19 months of $97 \%$ versus $91 \%(\mathrm{p}<0.001)$. The annual rate of progression to AP/BC appeared to be fairly constant in the first 4 years of treatment: $1.5 \%, 2.8 \%, 1.6 \%$ and $0.9 \%$; long-term follow up is awaited. ${ }^{13}$ Because of the remarkable remission rates with imatinib led to the crossover of most patients in the interferon alfa group to the imatinib group, long-term comparisons of survival for imatinib and interferon alfa are not possible. Analysis of the IRIS study shows that the Sokal score, which is based on age, spleen size, platelet and peripheral blood blast count, ${ }^{14}$ is well correlated with the likelihood of achieving complete cytogenetic response: $91 \%$ for low-, $84 \%$ for intermediate- and 69\% for highrisk patients. But patients who achieved a major molecular response at 12 months were also free from progression at 54 months, and none of these individuals had progressed to AP or BC, regardless of pretherapeutic Sokal risk group. ${ }^{9}$ Among patients lacking a complete cytogenetic response at 12 months, $28 \%$ had progressed by 54 months, and $11 \%$ to AP or BC. These data underscore the need for close monitoring for responses periodically once treatment with imatinib is initiated.

\section{High-dose imatinib}

Patients in accelerated phases of CML who were treated with imatinib at 400 or $600 \mathrm{mg} / \mathrm{d}$ were evaluated for hematologic and cytogenetic response, time to progression, survival and toxicity. In comparison with $400 \mathrm{mg}$, imatinib doses of $600 \mathrm{mg} / \mathrm{d}$ led to more cytogenetic responses (28\% compared to $16 \%)$, longer duration of response (79\% compared with $57 \%$ at 12 months), time to disease progression (67\% compared with $44 \%$ at 12 months), and overall survival (OS: 78\% compared with $65 \%$ at 12 months), with no clinically relevant increase in toxicity. ${ }^{9,15}$ Thus, a daily dose of $600 \mathrm{mg}$ was likely to be more effective than $400 \mathrm{mg}$ for $\mathrm{AP} / \mathrm{BC}$ patients.

Since at higher concentrations imatinib may inhibit more effectively BCR-ABL and some mutants, studies were initiated to test higher doses also in chronic phase. In patients with both prior hematologic and cytogenetic resistance to $400 \mathrm{mg}$ of imatinib daily, increasing the dose to $800 \mathrm{mg}$ resulted in a complete hematologic remission in $65 \%$ of patients and a complete cytogenetic response in $18 \%$ of treated patients. ${ }^{15}$ Compared with standard-dose imatinib, $800 \mathrm{mg}$ daily dose of imatinib was associated 
with significantly better complete cytogenetic response $(p=0.0005)$, major molecular response $(p=0.00001)$ and complete molecular response $(p=0.001)$ at the price of frequent myelosuppression. ${ }^{16}$ The role of high-dose imatinib (>400 mg daily) upfront in chronic phase patients with high-risk features is being evaluated in clinical studies and remains a category $2 \mathrm{~A}$ recommendation according to the National Comprehensive Cancer Care Network (NCCN) guidelines. ${ }^{17}$ Interim results of TOPS (Tyrosine Kinase Inhibitor Optimization and Selectivity), which compared $400 \mathrm{mg}$ with $800 \mathrm{mg}$, had shown that high-dose imatinib is associated with rapid major molecular responses, but this difference was not statistically significant from that achieved with standard-dose imatinib. ${ }^{18}$

\section{Late chronic phase and imatinib}

Patients who were in chronic phase, but resistant or intolerant to interferon alfa or who had been treated with conventional chemotherapy, are classified as late chronic phase. At 5 years of follow up in phase II trials, $69 \%$ of such patients treated with imatinib overall were free from progression to AP or BC. ${ }^{16,19,20}$ Long-term follow up (72 months) of late chronic phase patients from the Italian Cooperative Study Group on CML showed a complete cytogenetic response rate of $55 \%$ and major molecular response rate of $68 \% .{ }^{19}$ Overall the survival of cytogenetic responders was more than $90 \%$ and the PFS and OS of complete cytogenetic responders have remained superior after a median observation time of 6 years. Achievement of a complete cytogenetic response in late chronic phase is prognostically as important as in early stage chronic phase CML patients and was stable over the follow up period.

\section{Clinical results in AP and BC}

In the majority of patients with CML in $\mathrm{BC}$ and Ph-chromosome-positive acute lymphocytic leukemia (ALL), the leukemic clone remains at least partially dependent on
BCR-ABL for survival. BC (Table 1) is highly refractory to conventional induction therapy with chemotherapeutic drugs. A study conducted by Druker et al showed that imatinib was active with an overall response rate in the myeloid blast crisis of $55 \%$, and the rate of complete remission was $11 \%$. Sustained hematologic response and complete hematologic response was noted in $36 \%$ and $9 \%$ of previously untreated patients. Major cytogenetic response (16\%) and complete cytogenetic response $(7 \%)$ were markedly higher in patients treated with an initial imatinib dose of $600 \mathrm{mg}$ daily than in those given $400 \mathrm{mg}$ daily. ${ }^{21}$ The PFS and median survival of patients treated with imatinib in AP and BC were short; thus though imatinib remains a useful strategy even in advanced phases, these patients will benefit from combination therapies and stem cell transplantation. ${ }^{15}$

Response rates or the durability of responses in patients with lymphoid BC and those with $\mathrm{Ph}$-chromosome-positive ALL were similar in clinical studies. Overall response rate in patients with lymphoid BC or Ph-chromosome-positive ALL was $70 \%$, and $20 \%$ of patients had complete remissions, ${ }^{15}$ with early relapse in most patients.

\section{Adverse effects to imatinib}

Adverse events (Table 2) with imatinib are generally mild, with rare grade 3 or 4 toxicities. Myelosuppression (neutropenia, thrombocytopenia, anemia) is the most frequent adverse event ( $16.7 \%$ in the IRIS study). These are manageable with supportive therapy with growth factors and blood transfusions without the need for interruption of imatinib. Mild elevation in liver enzymes (5.3\%) and peripheral edema $(4.5 \%)$ were other notable side effects. Decreasing fluid intake or diuretics are helpful for managing fluid retention. Other side effects may include gastrointestinal intolerance, rash, myalgia, arthralgia and drug interactions due to inhibition of the P450 pathway. In smaller series, changes in bone and mineral metabolism and congestive heart failure were associated with imatinib treatment.

Table I Definition of chronic, accelerated and blastic phases of chronic myeloid leukemia by World Health Organization Criteria

\begin{tabular}{l}
\hline CML phase definition \\
\hline Chronic phase \\
Presence in peripheral blood or bone marrow of blasts $<10 \%$, basophils $<20 \%$, blasts and promyelocytes $<30 \%$, and platelets $\geq 100 \times 10 \% / L$ \\
Accelerated phase \\
Peripheral blood or bone marrow blasts of $10 \%-19 \%$, or blasts and promyelocytes $\geq 30 \%$, or basophils $\geq 20 \%$, or platelets $<100 \times 10 \% / L$ not related to \\
therapy, or chromosomal abnormalities other than the Ph chromosome, or progressive splenomegaly \\
Blast crisis \\
Presence of $>20 \%$ blasts in blood or bone marrow or evidence of extramedullary blast infiltration (except liver, spleen, or lymph nodes) or large \\
clusters of blasts in bone marrow biopsy
\end{tabular}


Table 2 Frequency of adverse effects among patients receiving imatinib for chronic myeloid leukemia

\begin{tabular}{llll}
\hline Dose/toxicity & Chronic phase (n 532) & Accelerated phase (n 235) & Blast phase (n 260) \\
\hline Dose & 100 & 33 & 14 \\
$400 \mathrm{mg} /$ day & 0 & 67 & 86 \\
$600 \mathrm{mg} /$ day & & & 69 \\
Side effects & 68 & 71 & 69 \\
Nausea & 56 & 71 & 26 \\
Edema & 50 & 37 & 41 \\
Cramps & 37 & 53 & 52 \\
Diarrhea & 30 & 55 & 34 \\
Vomiting & 39 & 43 & 26 \\
Rash & 30 & 29 & 28 \\
Headache & 31 & 36 & 24 \\
Fatigue & 30 & 29 & 63 \\
Arthralgia & 34 & 58 & 60 \\
Neutropenia & 17 & 43 & 51 \\
Thrombocytopenia & 5 & 39 & \\
Anemia & & &
\end{tabular}

Little information about possible teratogenicity of imatinib in human beings is available; use of contraception during imatinib treatment is recommended.

\section{Diagnostic evaluation and disease monitoring}

At diagnosis, cytogenetics, reverse transcription-polymerase chain reaction (RT-PCR), and fluorescence in situ hybridization (FISH) are performed in addition to complete blood count, bone marrow biopsy, staging and Sokal risk stratification (spleen size and extramedullary disease). Recommendations for follow up and monitoring are outlined in Table 3.

Bone marrow biopsies should be done at 6-month intervals until achievement of complete cytogenetic response, to determine disease status. Evaluation of bone marrow samples for cytogenetic abnormalities is essential to rule out clonal cytogenetic evolution during therapy, which identifies patients at high risk of relapse. Once complete cytogenetic response is achieved, monitoring can continue with quantitative RT-PCR (qPCR) from peripheral blood at 3-month intervals. Though few patients (around 5\% of patients) with cytogenetic responses have developed clonal cytogenetic abnormalities in Ph-negative cells, the significance of this finding is less clear. ${ }^{22-24}$ The risk of progression to myelodysplastic syndrome or acute myeloid leukemia from such abnormalities in $\mathrm{Ph}$-negative cells is rare and thus, outside of a research setting and in the absence of significant hematologic abnormalities such as cytopenias, dysplastic morphology or Ph-negative clonal cytogenetic abnormalities on preceding biopsies, regular bone marrow biopsies may not be indicated. For complete cytogenetic responders, quantitative PCR remains the mainstay of monitoring continued response to imatinib.

\section{Definition of failure of standard-dose imatinib}

Follow up of the IRIS study at 60 months indicates that $16 \%$ of patients failed therapy with imatinib and 7\% progressed to AP or BC. Presence of factors such as derivative chromosome 9 deletion, additional chromosome aberrations in $\mathrm{Ph}$-positive cells at diagnosis, less than major molecular response at 12 months, or any increase in transcription of BCR-ABL or other chromosomal aberrations may suggest that standard doses may not be adequate, thus warranting more close observation in this subset of patients. ${ }^{25-27}$ Imatinib failure is defined as no hematological response at 3 months, incomplete hematological response or no cytogenetic response at 6 months, less than partial cytogenetic response at 12 months, less than complete cytogenetic response at 18 months, loss of complete hematological or cytogenetic remission, or detection of highly resistant mutations of BCR-ABL at any time. Suboptimal response is defined as incomplete hematological response at 3 months, less than partial cytogenetic response at 6 months, less than complete cytogenetic response at 12 months, less than major molecular remission at 18 months, loss of major molecular response, mildly resistant mutations, or other chromosome abnormalities. It is imperative to follow patient responses 
Table 3 Monitoring patients on imatinib

\begin{tabular}{llll}
\hline & Hematologic response & Cytogenetic response & Molecular response \\
\hline Recommended monitoring & Every 2 weeks until complete & Every 6 months until complete & Every 3 months; mutational \\
interval & $\begin{array}{l}\text { response, then every } 3 \text { months } \\
\text { unless otherwise required }\end{array}$ & $\begin{array}{l}\text { response achieved and confirmed, } \\
\text { hence at least every } 12 \text { months }\end{array}$ & $\begin{array}{l}\text { analysis in case of failure, suboptimal } \\
\text { response, or transcript level increase }\end{array}$ \\
\hline
\end{tabular}

closely and recognize suboptimal response or treatment failure at the earliest to perform additional tests and make necessary adjustments to treatment regimen.

\section{Primary resistance}

Failure to achieve a complete hematologic response by 3 months, cytogenetic response by 6 months, or major cytogenetic response by 12 months after starting imatinib is referred to as imatinib treatment failure and warrants a change in therapy. Primary hematologic resistance is rare in chronic phase CML but primary cytogenetic resistance is evident in $15 \%$ to $25 \%$ of patients. Drug transporters play an important role in uptake and elimination via gastrointestinal tract of imatinib. They also determine intracellular drug concentration. Drug efflux proteins have been extensively studied, and their overexpression has been frequently implicated as a cause of resistance. In this regard, high expression of the ATP-binding cassette $(\mathrm{ABC})$ transporter is also known to decrease intracellular concentration of imatinib. ${ }^{28,29}$ Similarly low organic cation transporter-1 (OCT-1) activity may play a role in decreasing intracellular concentration of imatinib and predispose to resistance. ${ }^{28,29}$

\section{Secondary resistance}

Relapse or secondary resistance is defined as a loss of complete hematologic response, confirmed loss of partial or complete cytogenetic response, or a confirmed $30 \%$ increase of Ph-positive metaphases defined relapse. In one study, a single 2-fold rise of $B C R-A B L$ transcripts was highly predictive of mutations in the kinase domain of $B C R-A B L$ and subsequent relapse. For patients treated with BCR-ABL kinase inhibitor therapy, it is important to investigate by both mutation and cytogenetic analyses the cause of rising BCR-ABL levels.

Cytogenetic abnormalities in the $\mathrm{Ph}$-positive clone have been detected in $58 \%$ of imatinib-resistant patients. The detection of a second $\mathrm{Ph}$ chromosome indicates amplification of the $B C R-A B L$ gene, which has been reported as a mechanism of imatinib resistance with an overall frequency of about $18 \% .{ }^{25}$ Rising BCR-ABL levels may be also related to inconsistent therapy (poor compliance), which should be considered.

Among the mechanisms known for imatinib resistance, point mutations are emerging as the major cause of resistance. Mutations that involve direct contact points between imatinib and $\mathrm{Abl}$ may impair binding by steric hindrance. Later disease stages, older age, prior interferon therapy, development of clonal evolution, high-risk Sokal score at diagnosis, and failure to achieve a complete cytogenetic response with imatinib may be associated with mutations and resistance to imatinib therapy. ${ }^{30}$ Failure to achieve a complete cytogenetic response by 12 months of imatinib therapy was associated with the detection of mutations in $28 \%$ of patients. Thus, it is suggested that kinase domain mutational analysis should be performed when there is failure of response to imatinib or suboptimal response, and rising BCR-ABL levels.

Association between mutations in the P-loop of the BCR-ABL kinase domain and a poorer prognosis for patients who were treated with imatinib has been reported in some clinical studies ${ }^{31,32}$ but not in others. ${ }^{33}$ Kinase domain mutations detected at the P-loop and at residues T315 and M351 constitute $60 \%$ to $85 \%$ of all mutations tested. Other mechanisms of secondary resistance include activation of Src family of kinases and additional chromosomal abnormalities in Ph-positive cells

\section{Options for patients with a suboptimal response to standard-dose imatinib}

Any patient with a suboptimal response or signs of resistance should be considered for a clinical trial, primarily with an alternative Abl kinase inhibitor or dose escalation of imatinib, which will be effective in $30 \%$ to $50 \%$ of patients. ${ }^{34}$ However, these responses may last for a short time period and stem cell transplantation should be considered as an option for patients with low transplant risk and failure to achieve complete hematologic response.

Approximately $30 \%$ of chronic-phase patients who will not respond or inevitably relapse can be identified by close monitoring at diagnosis; they can be offered secondary tyrosine kinase inhibitors or transplantation at the outset. It is now known that Bcr-Abl remains the optimal target even 
at the time of relapse and has driven the search for alternative approaches to restore target inhibition. There is evidence in the majority of patients with acquired resistance of either increased expression of Bcr-Abl or, more frequently, mutations in the kinase domain of Bcr-Abl that interfere with drug binding.

Alternative Abl inhibitors that exhibit increased potency have been evaluated in phase I/II trials and have demonstrated very encouraging clinical activity. Nilotinib is approximately 20 -fold more potent than imatinib and dasatinib. Initially developed as a Src kinase inhibitor but also noted to be an extremely potent inhibitor of $\mathrm{Abl}$ (300-fold greater activity than imatinib), nilotinib is active against most imatinib-resistant kinase Abl mutants, with the notable exception of the T315I mutant. ${ }^{35,36}$ Responses in patients in AP were also impressive and appear to be mostly stable. Given these excellent results, treatment with an alternative Abl kinase inhibitor is probably the best drug therapy option available for patients with primary or acquired resistance to imatinib.

In summary, the recommendation for patients with mutations at Y253, E255 and T315 is allogeneic transplant, another kinase inhibitor, or investigational treatments, as these suggest high-level imatinib resitance and failure. ${ }^{37}$ Others such as mutations at residues M244, F317 and M351 may respond to an increased imatinib dose, as they indicate low level resistance. ${ }^{37}$ Mutations in addition to the above may have clinical significance, although much more remains to be learnt.

\section{Imatinib vs other second-generation tyrosine kinase inhibitors}

Comparative studies of imatinib and dasatinib or bosutinib or combinations of tyrosine kinase inhibitors are ongoing. Some of these trials are outlined in Table 4. A recent study reported by Kantarjian et al of high dose imatinib (800 mg) vs dasatinib $70 \mathrm{mg}$ for patients who had failed to respond to standard dose imatinib showed that the responses were comparable between two groups, suggesting that increasing the dose of imatinib is still a reasonable option. ${ }^{38}$

\section{Clonal cytogenetic abnormalities on imatinib}

$\mathrm{Ph}$-chromosome-negative hematopoiesis consists of cells derived from nonleukemic hematopoietic stem cells. Studies have described clonal cytogenetic abnormalities in Ph-negative cells from some patients who had a cytogenetic response to imatinib. In various studies, the frequency of such patients ranged from $2 \%$ to $15 \% .^{23,24}$ Some patients with typical chromosomal abnormalities had developed acute myeloid leukemia or myelodysplastic syndromes others have done well. ${ }^{23}$ Due to the fact that the available data are limited, the overall prognosis for such patients is unknown.

\section{Role of allogeneic transplantion in imatinib era}

Stem cell transplantation is the only treatment modality with the potential for cure in CML. While long-term data are still not available, because the likelihood of disease progression to AP or BC during the first year of imatinib therapy is only $1.5 \%$ and the transplant-related mortality during the first 12 months is $10 \%$ to $20 \%$, imatinib therapy is warranted as first choice in all patients.

NCCN recommends evaluation for stem cell transplantation for all patients who do not achieve a cytogenetic response at 6 months following imatinib therapy or have cytogenetic relapse at 12 or 18 months after achieving initial hematologic remission, achieve partial cytogenetic response at 18 months, and who have T3151 mutation and have failed on imatinib, dasatinib or nalotinib.

In contrast to its use in the chronic phase, there is a welldefined role for stem cell transplantation in the management

Table 4 Active imatinib/combination phase III randomized studies

Study site randomization

Germany/Switzerland Imatinib \pm interferon alfa or cytarabine vs interferon followed by stem cell transplant

France Imatinib $400 \mathrm{mg}$ vs imatinib $400 \mathrm{mg}$ + cytarabine vs imatinib $400 \mathrm{mg}$ + peginterferon alfa-2a vs imatinib $600 \mathrm{mg}$

Austria Imatinib $800 \mathrm{mg}$ followed by imatinib $400 \mathrm{mg}$ compared with a continuous treatment with imatinib $400 \mathrm{mg}$

Israel Imatinib vs imatinib and peginterferon after complete cytogenetic response with imatinib therapy

USA/UK Nilotinib $300 \mathrm{mg}$ vs $400 \mathrm{mg}$ twice daily vs imatinib $400 \mathrm{mg}$

Multinational Dasatinib vs imatinib

Multinational Bosutinib vs imatinib

OncoTargets and Therapy 2009:2 
of advanced CML, when imatinib responses tend to be transient and short lived. Thus, in patients who progressed to $\mathrm{AP} / \mathrm{BC}$ on imatinib, the novel $\mathrm{Abl}$ kinase inhibitors will likely induce transient remission and serve as a bridge to stem cell transplantation.

\section{Imatinib combinations}

A multicentric, phase II study of imatinib $400 \mathrm{mg}$ daily and pegylated interferon alfa in the treatment of 76 early chronic phase $\mathrm{Ph}$-positive CML patients confirmed the excellent response to front-line imatinib and the stability of the complete cytogenetic response. Any possible additional benefit of the combination with interferon alfa remained uncertain due to poor compliance to interferon.

The efficacy of the triple combination regimen with interferon alfa, ara-C, HHT and sequential imatinib therapy were evaluated in newly diagnosed $\mathrm{Ph}$-positive CML, which resulted in improved estimated 5-year survival rates.

More combinations with imatinib have been studied but randomized clinical trials are ongoing around the world, some of which are outlined in Table 4.

\section{Summary}

Availability of imatinib as an accepted targeted therapy in newly diagnosed patients has changed the treatment paradigm in CML. Yet identification of newer mechanisms of resistance to imatinib and various molecular mutations have made overall management of CML complex. The majority of CML patients in chronic phase achieve excellent and durable responses with standard-dose imatinib, but since resistance can develop at any time during treatment, close monitoring, including quantitative PCR, is crucial. Newer Abl kinase inhibitors, such as nilotinib and dasatinib, with activity against mutant $\mathrm{Bcr}-\mathrm{Abl}$, are the most promising drug therapy for imatinib failure in chronic phase. Eligible patients with AP or BC should undergo allogeneic transplant, with imatinib or alternative tyrosine kinase inhibitor therapies used for bridging, as responses are short lived (3 months or so). Pre-treatment with imatinib does not seem to affect transplant outcome at any phase. ${ }^{39}$

\section{Future research}

Currently, we are unable to identify up-front a small subgroup of high-risk patients who will not respond well to tyrosine kinase inhibition (primary resistance). Systematic screening for kinase domain mutations on patients before imatinib or to predict response or secondary resistance to imatinib is not recommended as it may not be cost-effective. Higher doses of imatinib or combination of tyrosine kinase inhinitors in chronic phase are not clear awaiting clinical trial results, and whether earlier achievement of these responses is important for progression-free survival remains largely unknown. It has not been resolved whether patients with undetectable BCR-ABL will be able to cease imatinib treatment and maintain response. A recent report indicates that this may be the case for selected patients, at least in the short term. ${ }^{40}$ Additional mutations that may play a role in mediating secondary resistance to imatinib are not yet known and are being studied. In short, development of imatinib has led to better understanding of CML as a disease that has in turn led to development of more potent targeted therapies such as the second- and third-generation tyrosine kinase imhibitors to overcome resistance and further improve clinical outcome.

\section{Disclosures}

The authors declare no conflicts of interest.

\section{References}

1. Jemal A, Siegel R, Ward E, et al. Cancer Statistics. CA Cancer J Clin. 2008;58(2):71-96.

2. Nowell PC, Hungerford DA. A minute chromosome in human granulocytic leukemia. Science. 1960;132:1497-1497.

3. Sawyers CL. Medical Progress. Chronic myeloid leukemia. $N$ Engl J Med. 1999;340:1330-1340.

4. Rowley JD. A new consistent chromosomal abnormality in chronic myelogenous leukaemia identified by quinacrine fluorescence and Giemsa staining. Nature. 1973;243:290-293.

5. Shtivelman E, Lifshitz B, Gale RP, Canaani E. Fused transcript of abl and bcr genes in chronic myelogenous leukaemia. Nature. 1985;315:550-554.

6. Daley GQ, Van Etten RA, Baltimore D. Induction of chronic myelogenous leukemia in mice by the P210bcr/abl gene of the Philadelphia chromosome. Science. 1990;247:824-830.

7. Kelliher MA, McLaughlin J, Witte ON, Rosenberg N. Induction of a chronic myelogenous leukemia-like syndrome in mice with $\mathrm{v}$-abl and BCR-ABL. Proc Natl Acad Sci U S A. 1990;87:6649-6653.

8. Druker BJ, Tamura S, Buchdunger E, et al. Effects of a selective inhibitor of the Abl tyrosine kinase on the growth of Bcr-Abl positive cells. Nat Med. 1996;2:561-566.

9. Druker BJ, Guilhot F, O'Brien SG, et al. Five-year follow-up of patients receiving imatinib for chronic myeloid leukemia. $N$ Engl $J$ Med. 2006;355:2408-2417.

10. Faderl S, Talpaz, Estrov Z, Kantarjian HM, et al. Chronic myelogenous leukemia. biology and therapy. Ann Int Med. 1999;131:207-219.

11. Baccarani M, Saglio G, Goldman J, et al. Evolving concepts in the management of chronic myeloid leukemia: recommendations from an expert panel on behalf of the European LeukemiaNet. Blood. 2006;108(6): 1809-1820.

12. O'Brien SG, Guilhot F, Larson RA, et al. Imatinib compared with interferon and low-dose cytarabine for newly diagnosed chronic-phase chronic myeloid leukemia. N Engl J Med. 2003;348:994-1004.

13. Simonsson B, on behalf of the IRIS study group. Beneficial effects of cytogenetic and molecular response on long term outcome in patients with newly diagnosed chronic myeloid leukemia in chronic phase (CMLCP) treated with Imatinib (IM): update from the IRIS study [abstract]. Blood. 2005;106:52a. Abstract 166.

14. Sokal JE, Cox EB, Baccarani M, et al. Prognostic discrimination in "good-risk" chronic granulocytic leukemia. Blood. 1984;63:789-799. 
15. Talpaz M, Silver RT, Druker BJ, et al. Imatinib induces durable hematologic and cytogenetic responses in patients with accelerated phase chronic myeloid leukemia: results of a phase 2 study. Blood. 2002;99:1928-1937.

16. Kantarjian, Talpaz M, O'Brien S, et al. High-dose imatinib mesylate therapy in newly diagnosed Philadelphia chromosome-positive chronic phase chronic myeloid leukemia. Blood. 2004;103(8):2873-2778.

17. Chronic myelogenous leukemia. National Comprehensive Care Network Practice guidelines in oncology. Version 2. 2009.

18. Cortes J, Baccarani M, Guilhot F, et al. First report of the TOPS study: A randomized phase III study of $400 \mathrm{mg}$ vs $800 \mathrm{mg}$ Imatinib in patients with newly diagnosed, previously untreated CML, in chronic phase using molecular end points [abstract]. 13th Congress of European Hematological Association. Abstract 402.

19. Palandri F. Long-term outcome of complete cytogenetic responders after imatinib $400 \mathrm{mg}$ in late chronic phase, philadelphia-positive chronic myeloid leukemia: the GIMEMA Working Party on CML. J Clin Oncol. 2008;26(1):106-111.

20. Silver RT, Talpaz M, Sawyers CL, et al. Four years of follow-up of 1027 patients with late chronic phase (L-CP), accelerated phase (AP), or blast crisis $(\mathrm{BC})$ chronic myeloid leukemia $(\mathrm{CML})$ treated with imatinib in three large phase II trials [abstract]. Blood. 2004;104:11a Abstract 23.

21. Sawyers CL, Hochhaus A, Feldman E, et al. Imatinib induces hematologic and cytogenetic responses in patients with chronic myelogenous leukemia in myeloid blast crisis: results of a phase II study. Blood. 2002;99(10):3530-3539.

22. Medina J, Kantarjian H, Talpaz M, et al. Chromosomal abnormalities in Philadelphia chromosome-negative metaphases appearing during imatinib mesylate therapy in patients with Philadelphia chromosomepositive chronic myelogenous leukemia in chronic phase. Cancer. 2003;98(9):1905-1911.

23. Loriaux M, Deininger M. Clonal cytogenetic abnormalities in Philadelphia chromosome negative cells in chronic myeloid leukemia patients treated with imatinib. Leuk Lymphoma. 2004;45:2197-2203.

24. Terre C, Eclache V, Rousselot P, et al. Report of 34 patients with clonal chromosomal abnormalities in Philadelphia-negative cells during imatinib treatment of Philadelphia-positive chronic myeloid leukemia. Leukemia. 2004;18:1340-1346.

25. Melo JV, Chuah C. Resistance to imatinib mesylate in chronic myeloid leukaemia. Cancer Lett. 2007;249:121-132.

26. Cervantes F, Hernandez-Boluda JC, Steegmann JL, et al; Imatinib mesylate therapy of chronic phase chronic myeloid leukemia resistant or intolerant to interferon: Results and prognostic factors for response and progressionfree survival in 150 patients. Haematologica. 2003;88:1117-1122.

27. Huntly BJP, Reid AG, Bench AJ, Campbell LJ, Telford N, Shepherd P Deletions of the derivative chromosome 9 occur at the time of the Philadelphia translocation and provide a powerful and independent prognostic indicator in chronic myeloid leukemia. Blood. 2001;98:1732-1738.
28. White DL, Saunders VA, Dang P, et al. OCT-1 mediated influx is a key determinant of the intracellular uptake of imatinib but not nilotinib (AMN 107): reduced OCT 1 activity is the cause of low in vitro sensitivity to imatinib. Blood. 2006;108(2):697-704.

29. White DL, Saunders VA, Dang P, et al. Most CML patients who have a sub optimal response to imatinib have low OCT-1 activity; higher doses of imatinib may overcome the negative impact of low OCT-1 activity. Blood. 2007;110(12):4064-4072.

30. Jabbour E, Kantarjian H, Jones D, et al. Frequency and clinical significance of BCR-ABL mutations in patients with chronic myeloid leukemia treated with imatinib mesylate. Leukemia. 2006;20:1767-1773.

31. Nicolini FE, Corm S, Le QH, et al. Mutation status and clinical outcome of 89 imatinib mesylate-resistant chronic myelogenous leukemia patients: a retrospective analysis from the French intergroup of CML. Leukemia. 2006;20:1061-1066.

32. Branford S, Rudzki Z, Walsh S, et al. Detection of BCR-ABL mutations in patients with CML treated with imatinib is virtually always accompanied by clinical resistance, and mutations in the ATP phosphate-binding loop (P-loop) are associated with a poor prognosis. Blood. 2003;102:276-283.

33. Shah NP, Tran C, Lee FY, Chen P, Norris D, Sawyers CL. Overriding imatinib resistance with a novel ABL kinase inhibitor. Science. 2004;305:399-401.

34. Kantarjian HM, Talpaz M, O'Brien S, et al. Dose escalation of imatinib mesylate can overcome resistance to standard-dose therapy in patients with chronic myelogenous leukemia. Blood. 2003;101:473-475.

35. Talpaz M, Shah NP, Kantarjian H, et al. Dasatinib in imatinibresistant Philadelphia chromosome-positive leukemias. $N$ Engl $J$ Med. 2006;354:2531-2541.

36. Kantarjian H, Giles F, Wunderle L, et al. Nilotinib in imatinib-resistant CML and Philadelphia chromosome-positive ALL. $N$ Engl J Med. 2006;354:2542-2551.

37. Baccarani M, Saglio G, Goldman J, et al. Evolving concepts in the management of chronic myeloid leukemia: recommendations from an expert panel on behalf of the European LeukemiaNet. Blood. 2006;108:1809-1820.

38. Kantarjian H, Schiffer C, Jones D, Cortes J, et al. Monitoring the response and course of chronic myeloid leukemia in the modern era of BCR-ABL tyrosine kinase inhibitors: practical advice on the use and interpretation of monitoring methods. Blood. 2008;111(4):1774-1780.

39. Oehler VG, Gooley T, Snyder DS, et al. The effects of imatinib mesylate treatment before allogeneic transplantation for chronic myeloid leukemia. Blood. 2007;109:1782-1789.

40. Rousselot P, Huguet F, Rea D, et al. Imatinib mesylate discontinuation in patients with chronic myelogenous leukemia in complete molecular remission for more than 2 years. Blood. 2007;109:58-60.

41. Savage DG, Antman KH. Imatinib mesylate - a new oral targeted therapy. N Engl J Med. 2002;346:683-693. 
